\title{
The genetic value of sons and daughters
}

\author{
Steven A. Frank
}

Division of Biological Sciences, University of Michigan, Ann Arbor, MI 48109-1048 U.S.A.

Comments are presented on the recent paper by Bulmer (1986) concerning the sex ratio.

Bulmer (1986) has recently studied sex ratio evolution under the island migration model. His study was motivated by my statement that as genetic differentiation among isolated patches increases, the predicted proportion of males declines linearly with $\boldsymbol{P}_{d t}$, Wright's (1969) measure for differentiation among demes (Frank, 1985, 1986). Bulmer's results show, under the assumptions of his particular model, that the sex ratio is independent of the migration rate for a diploid organism. Since $P_{d t}$ declines towards zero as the migration rate declines-no migration leads to complete fixation within demes-Bulmer's result suggests that the sex ratio is independent of $P_{d r}$. Here I show that different model assumptions are the cause underlying the discrepancy between our results. I also derive an analytic solution for the scenario studied numerically by Bulmer, by applying the genetic value methodology developed in Frank (1986).

The scenario Bulmer studied is characterised by the following assumptions. (i) There are a large number of discrete patches in the population. (ii) In each discrete, non-overlapping generation, mating occurs randomly within patches, and there is no mating between individuals born in different patches. (iii) A fraction $m$ of the mated females disperse and settle randomly in each generation, while $1-m$ of the mated females remain in their natal patch. (iv) Within each patch, $N$ mated females are chosen randomly from among dispersers and non-dispersers, and these females reproduce to form the next generation.

I will first consider a model of a diploid organism with maternal control of the sex ratio. Other types of genetic control studied by Bulmer will be discussed later. To obtain the ESS sex ratio (proportion of males) as functions of $m$ and $N$ by using hierarchical selection theory, all that we need is a statement about the expected number of grandprogeny of an individual, sometimes called the "adaptive function" (Frank, 1986), and the assumption that the sex ratio is controlled at a single locus with additive gene action. Let there be two sex ratio phenotypes: an $a$-type with sex ratio $r_{a}$, and a $b$-type with sex ratio $r_{b}$; let the average sex ratio in the deme be $r_{d}$; and let the average sex ratio in the population be $r$. To obtain the expected number of grandprogeny, begin by calling $z=(1-m)\left(1-r_{d}\right)+m(1-r)$ proportional to the expected number of mated females in the natal patch, after migration, that compete for a fixed number of breeding spots, $N$. The expected number of competing females in a random patch is proportional to $1-r$. Therefore the expected number of grandprogeny through daughters for an $a$-type is proportional to

$$
\left(1-r_{a}\right)\left(\frac{m}{1-r}+\frac{1-m}{z}\right)
$$

The expected number of inseminations by males of an $a$-type is proportional to $\left(r_{a} / r_{d}\right)\left(1-r_{d}\right)$, so the expected number of grandprogeny through sons is

$$
\left(r_{a} / r_{d}\right)\left(1-r_{d}\right)\left(\frac{m}{1-r}+\frac{1-m}{z}\right) .
$$

The expected number of grandprogeny (the adaptive function) for an $a$-type in a deme with sex ratio $r_{d}$ is,

$$
\begin{aligned}
w_{d a}= & \left(\frac{r_{a}\left(1-r_{d}\right)}{r_{d}}\right)\left(\frac{m}{1-r}+\frac{1-m}{z}\right) \\
& +\left(1-r_{a}\right)\left(\frac{m}{1-r}+\frac{1-m}{z}\right) .
\end{aligned}
$$

By applying hierarchical selection theory, as explained in detail in Frank (1986), the ESS proportion of males is,

$$
r^{*}=\frac{P_{d t}}{D+(2-D) P_{d t}}
$$

where $D=2 m(2-m)$, and $P_{d t}$ is a form of Wright's (1969) index of panmixia, which is a measure of the additive genotypic variance within demes divided by the additive genotypic variance in the population. As described by Wright, $\rho=1-P_{d t}$ is a measure of the genotypic correlation among individuals within the deme. Using this identity, we obtain,

$$
r^{*}=\frac{1-\rho}{2-\rho(2-D)}=\frac{1-\rho}{2\left[1-(1-m)^{2} \rho\right]} .
$$


Since $r^{*}$ is proportional to the allocation in males divided by the allocation in males plus the allocation in females, it is possible to represent $r^{*}$ in a ratio form (allocation to males: allocation to females)

$$
1-\rho: 1-(1-m)^{2} \rho+\left[1-(1-m)^{2}\right] \rho .
$$

If a meaning can be assigned to $\rho$, then we will be in a position both to understand the causal forces affecting the sex ratio, and to write down an analytic solution for this scenario. One choice for $\rho$ is the regression coefficient of relatedness of the genotype that controls the sex ratio onto males or females, depending on the context (to be discussed shortly). There are three rationalisations for this choice. (i) For scenarios studied in Frank (1986), and for the haploid and diploid models of Bulmer (1986), the regression coefficient gives results that agree with the special cases studied by traditional methods. (ii) Regression implies causal mechanisms for sex ratios that make a good deal of intuitive sense (see below). (iii) The use of an adaptive function, $w_{d a}$ above, is essentially a statement about competition among clones: $a$-types and $b$-types. The elegance of regression is that it translates different ploidy levels and forms of sexuality into a common currency-numbers of genes identical by descent (ibd) (Hamilton, 1972). Covariance among additive genotypic values is a related and often preferable currency to genes ibd (Seger, 1981), but I will stick with ibd here since it is easier to use in verbal descriptions.

I will consider just one case in detail here-a diploid organism with maternal control of the sex ratio. It is simplest first to assign regression interpretations for each of the $\rho$ 's in equation (2), and then to rearrange the expression to clarify the meaning of the result. Equation (2) in regression notation is,

$$
1-b_{m \rightarrow \delta^{+}}: 1-(1-m)^{2} b_{m \rightarrow q}+\left[1-(1-m)^{2}\right] b_{m \rightarrow \delta^{+}}
$$

where $b_{m \rightarrow 0^{*}}$ is the regression of mother on male progeny in the group, and $b_{m \rightarrow q}$ is the regression of mother on female progeny in the group before migration. Regression is the number of genes ibd in another individual divided by the number of genes ibd in one's own progeny (Hamilton, 1972). If $F$ is the probability that uniting gametes are ibd, then a mother can expect $(1 / 2)(1+3 F)$ genes ibd in her own offspring (either male or female), and can expect $2 F$ genes ibd in a randomly chosen progeny (male or female), hence $b_{m \rightarrow \delta^{*}}=b_{m \rightarrow q}=$
$2 F /[(1 / 2)(1+3 F)]$. Substituting into equation (3) and multiplying through by $(1 / 2)(1+3 F)$ gives,

$$
\begin{gathered}
(1 / 2)(1+3 F)-2 F:(1 / 2)(1+3 F)-(1-m)^{2}(2 F) \\
+\left[1-(1-m)^{2}\right](2 F) .
\end{gathered}
$$

The left side can now be read as: the genetic value of a son is the (expected) number of genes ibd from the mother in a son, $(1 / 2)(1+3 F)$, minus the number of genes ibd from the mother, $2 \mathrm{~F}$, that a son interferes with when competing for access to mates. On the right side, the genetic value of a daughter is: (i) the number of genes ibd, (ii) minus the number of genes ibd that a daughter interferes with when competing for access to a fixed number of breeding spaces-the probability that two females interacting are both from the same patch, $(1-m)^{2}$, multiplied by the expected number of genes ibd from the mother that are in a daughter's competitor from the same patch, $2 F$, plus (iii) the number of genes ibd that a daughter obtains from her mates and transmits to the following generation without interfering with ibd genes-the probability that the female is not involved in a competitive interaction with an individual from her natal patch, $\left[1-(1-m)^{2}\right]$, multiplied by the number of ibd genes from mates, $2 F$.

$F$ can be obtained by the usual recurrence techniques (Wright, 1969). Defining $F_{g}$ as the correlation of genes in the $g$ th generation,

$$
\begin{aligned}
F_{g}= & 1 / 4 N+(3 / 4 N) F_{g-1} \\
& +(1-m)^{2}[(N-1) / N] F_{g-1}
\end{aligned}
$$

and setting $F_{g}=F_{g-1}$ to obtain the steady-state correlation yields,

$$
F=\frac{1}{4 N-3-4(N-1)(1-m)^{2}} .
$$

Therefore, the value for $b_{m \rightarrow \delta^{\star}}=b_{m \rightarrow \uparrow}$ is,

$$
b=\frac{1}{N-(N-1)(1-m)^{2}} \text {. }
$$

Substituting into equation (1) yields the ESS $r^{*}=$ $(N-1) / 2 N$, for $0<m \leqq 1$, which agrees with Bulmer's numerical results for $N=2$, and for his haploid results for $N=2,3$, and 4. [For the haploid case, $b$ is the same as in equation (5)].

My results here agree with Bulmer's conclusion that the sex ratio is independent of the migration rate in an island model. The discrepancy with Frank $(1985,1986)$ noted by Bulmer is caused by the explicit assumption in my earlier papers that there is no limit on resources available to females, 
in contrast to Bulmer's fixed number of breeding sites. With no competition among females, the sex ratio is of the form $1-b_{m \rightarrow \delta}: 1+b_{m \rightarrow \delta}$ (Frank, 1986). Bulmer conjectured that the ESS under his scenario is independent of migration rate because of the balancing forces of increased competition among males against increased competition among females. The analysis here shows that this is partly correct, but that the fate of genes ibd from females' mates must also be considered [see equation (3)].

Bulmer obtained a number of other interesting results. For example, in a haploid model in which all of the males and none of the females disperse before mating, he found that the ESS was more female biased when fathers control the sex ratio than when mothers control the sex ratio. In this case the ESS is of the form $1: 1-b_{c \rightarrow q}$, where $b_{c \rightarrow q}$ is the regression of controlling genotype on female progeny. It is clear that a father will only be related to his daughters and no other females, since he is a newly arrived immigrant. So, the regression of father on female progeny will be $1 / N$. When a mother controls the sex ratio, she is of course related to her own daughters, but in addition there is a chance that other mothers in the group are related to her, so her regression on females is greater than $1 / N$. For this haploid case with mating after dispersal, it is straightforward to obtain an analytic solution for any amount of male and female migration under either mother or father control (see Appendix).

Another interesting set of results by Bulmer is the haplodiploid case, with mating before a fraction $m$ of the already mated females disperse. Haplodiploidy is a challenge for adaptive functions like the one used above, since these functions count number of grandprogeny. Hamilton (1979) conjectured that the adaptive function approach can be saved by simply weighting the number of grandprogeny through sons by the number of genes ibd in the son from the controlling genotype, and likewise weighting grandprogeny through daughters by the number of genes ibd in daughters (see Frank, 1986, for details). When all the females disperse after mating $(m=1)$ and there is maternal control, the genetic value technique yields $(1 / 2)(1+F)-F:(1 / 2)(1+3 F)+F$. Again, the genetic value of a son is the number of genes ibd minus the number interfered with, and the value of a daughter is the number of genes ibd plus the number obtained from mates. With random settling, $(m=1), F=1 /(4 N-3)$, and substituting in gives the standard result reported by Hamilton (1979) and Taylor and Bulmer (1980).
Acknowledgements Many of the ideas presented here have been stimulated and sharpened by the work of Michael Bulmer, and by numerous conversations with him while I was visiting Oxford. I thank W. D. Hamilton for generously arranging my visit to Oxford, where I completed the work presented here. This work was supported by awards 1-T32-GM-07544-07 and R01-GM-32589 from the National Institute of General Medical Sciences.

\section{REFERENCES}

BULMER, M. G. 1986. Sex ratio theory in geographically structured populations. Heredity, 56, 69-73.

FRANK, S. A. 1985. Hierarchical selection theory and sex ratios. II. On applying the theory, and a test with fig wasps. Evolution, 39, 949-964.

FRANK, S. A. 1986. Hierarchical selection theory and sex ratios. I. General solutions for structured populations. Theoret. Popul. Biol, in press.

HAMiLton, w. D. 1972. Altruism and related phenomena, mainly in the social insects. Ann. Rev. Ecol. Syst. 3, 192-232.

HAMILTON, W. D. 1979. Wingless and fighting males in fig wasps and other insects. In Reproductive Competition and Sexual Selection in Insects (M. S. Blum and N. A. Blum eds), pp. 167-220. Academic Press, New York.

SEGER, J. 1981. Kinship and covariance. J. Theoret. Biol., 91, 191-213.

TAYLOR, P. D. AND BULMER, M. G. 1980. Local mate competition and the sex ratio. J. Theoret. Biol., 86, 409-419.

WRIGHT, S. 1969. Evolution and the Genetics of Populations, Vol. 2, University of Chicago Press, Chicago.

\section{APPENDIX}

The derivation of the ESS sex ratio is presented for the following scenario: (i) The genetic system is haploid. (ii) The offspring disperse before mating. Males disperse with probability $m_{\delta}$, and females disperse with probability $m_{\text {o }}$. (iii) Control of the sex ratio can be by either the mother or father. In order to develop a general solution for either case, let $c$ denote the controlling genotype, where $c=\delta$ for paternal control, and $c=q$ for maternal control.

Following the general approach outlined in this paper, the genetic value of sons to daughters is,

$$
1-\left(1-m_{\delta}\right)^{2} b_{c \rightarrow \delta}: 1-\left(1-m_{\odot}\right)^{2} b_{c \rightarrow \uparrow},
$$

where $b_{c \rightarrow \delta}$ and $b_{c \rightarrow q}$ are the regressions of controlling genotype on males and females of the progeny generation before dispersal. Since the genetic system is haploid, the expected number of alleles ibd in a male or female progeny with respect to some arbitrary allele is the same as the frequency of alleles ibd in the composite formed by the offspring's mother plus the sperm she obtains from her mate. So $b_{c \rightarrow 0}$ and $b_{c \rightarrow q}$ are both equal to $b_{c \rightarrow \text { comp }}$, where comp refers to mated composites 
in the parental generation. With this notation we can apply the definition of regression (Hamilton, 1972) and obtain

$$
b_{c \rightarrow \text { comp }}=\frac{F_{c \leftrightarrow c o m p}}{(1 / 2)\left(1+F_{\AA^{\prime}}\right)},
$$

where $F$ is now generalised to describe the correlation between alleles chosen randomly from the two groups indexed by the subscripts on $F$. Substituting (A2) into (A1) and rearranging, we obtain the ESS sex ratio

$$
r^{*}=\frac{1+F_{q}-2\left(1-m_{\delta}\right)^{2} F_{c \leftrightarrow c o m p}}{2+2 F_{q}-2\left(\left(1-m_{\delta}\right)^{2}+\left(1-m_{\wp}\right)^{2}\right) F_{c \leftrightarrow \text { comp }}} .
$$

To obtain a solution we need the following recurrence relations

$$
\begin{aligned}
& F_{\zeta^{\prime}}=\left(1-m_{\delta}\right)\left(1-m_{\varnothing}\right) K \\
& F_{\wp \wp}=\left(1-m_{\wp}\right)^{2} K \\
& F_{\text {ठす }}=\left(1-m_{\text {ठ }}\right)^{2} K
\end{aligned}
$$

Note added in proof.-The use of regression coefficients for $\rho$ is intended as a conjecture, for which there are both good rationalisations and good circumstantial evidence. Peter D. Taylor (submitted) had independently and rigourously derived results analogous to my genetic value methodology that also use

$$
\begin{aligned}
& K=(1 / 2 N)\left(1+F_{\phi}\right) \\
& +((N-1) / 4 N)\left(F_{\varnothing \varnothing}+2 F_{\zeta}+F_{\delta す}\right) \\
& F_{c \leftrightarrow \text { comp }}=(1 / 2 N)\left(1+F_{q^{\prime}}\right) \\
& +((N-1) / 2 N)\left(F_{c c}+F_{Q^{3}}\right) \text {. }
\end{aligned}
$$

Solving for the correlation between mates, which is the usual fixation index, one obtains

$$
\begin{gathered}
F_{\oint^{*}}=2\left(1-m_{\delta}\right)\left(1-m_{\wp}\right) / Q \\
Q=4 N-2 N\left(1-m_{\delta}\right)\left(1-m_{\wp}\right)-(N-1) \\
\times\left(\left(1-m_{\delta}\right)^{2}+\left(1-m_{\wp}\right)^{2}\right) .
\end{gathered}
$$

To illustrate with some special cases, suppose that control is maternal, $c=q$, then

$$
F_{\wp \rightarrow \text { comp }}=\frac{4 N+(N-1)\left(\left(1-m_{q}\right)^{2}-\left(1-m_{\delta}\right)^{2}\right)}{2 N Q} \text {. }
$$

If $m_{\odot}=1$ and $m_{\delta}=0$, then substituting (A5) and (A4) into (A3) yields $r^{*}=(N-1) /(2 N-1)$, and if $m_{q}=0$ and $m_{\delta}=1$, then $r^{*}=$ $N(3 N+1) /\left(6 N^{2}-3 N+1\right)$, which agrees with Bulmer's (1986) numerical results.

Hamilton-type regression (or pedigree) coefficients. Taylor has also proved a general theorem that formally links exact genetic models with Price covariance models (hierarchical selection), and with inclusive fitness models based on regression coefficients. 